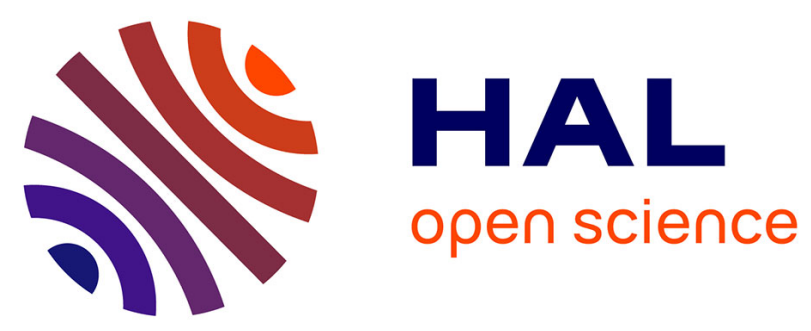

\title{
Design of a new parallel haptic device for desktop applications
}

\author{
Florian Gosselin, Jean-Pierre Martins, C. Bidard, Claude Andriot, Julien \\ Brisset
}

\section{- To cite this version:}

Florian Gosselin, Jean-Pierre Martins, C. Bidard, Claude Andriot, Julien Brisset. Design of a new parallel haptic device for desktop applications. First Joint Eurohaptics Conference and Symposium on Haptic Interfaces for Virtual Environment and Teleoperator Systems, IEEE, Mar 2005, Pisa, Italy. pp.189-194, 10.1109/WHC.2005.33 . cea-03254587

\section{HAL Id: cea-03254587 https://hal-cea.archives-ouvertes.fr/cea-03254587}

Submitted on 8 Jun 2021

HAL is a multi-disciplinary open access archive for the deposit and dissemination of scientific research documents, whether they are published or not. The documents may come from teaching and research institutions in France or abroad, or from public or private research centers.
L'archive ouverte pluridisciplinaire HAL, est destinée au dépôt et à la diffusion de documents scientifiques de niveau recherche, publiés ou non, émanant des établissements d'enseignement et de recherche français ou étrangers, des laboratoires publics ou privés. 


\title{
Design of a New Parallel Haptic Device for Desktop Applications
}

\author{
Florian GOSSELIN Jean-Pierre MARTINS \\ Catherine BIDARD Claude ANDRIOT Julien BRISSET \\ CEA LIST, Route du Panorama BP6, 92265 Fontenay aux Roses Cedex, France \\ E-mail: florian.gosselin, jp.martins, catherine.bidard, claude.andriot, julien.brisset@cea.fr
}

\begin{abstract}
In this paper, we present a new six degrees of freedom haptic device developed at CEA-LIST for desktop applications emphasizing quick and precise manipulation. This device relies on a light parallel architecture connecting the base of the robot to the mobile platform manipulated by the user. It is dimensioned and optimized to fit design requirements associated with Computer Aided Design or virtual sculpting. The design methodology relies on a geometric and static optimization which takes into account technological constraints associated with the main off the shelf components. The control scheme of this device is also described. Finally, feedback obtained from first integration tests are presented.
\end{abstract}

\section{Introduction}

Based on its long experience in remote handling technology [1] [2] which share the same requirements, the CEA-LIST recently launched several developments in haptics such as large workspace input devices for scale one applications[3], high fidelity master arms for telesurgery [4] or wearable haptic devices allowing fine manipulation with thumb and index fingers [5]. This article introduces another new input device dedicated to CAD like desktop applications.

Section 2 presents the design drivers associated with this kind of applications. Section 3 introduces the parallel architecture which is used for the design of the haptic interface. Section 4 presents the dimensioning and optimization procedure used to obtain the final design exposed in section 5. Section 6 introduces the control scheme used for the purpose of coupling the device with a virtual reality application. Finally, section 7 presents the feedback obtained from the first integration tests.

\section{Design drivers}

Whether considering virtual reality, telesurgery or remote handling, a 'good' input device must be 'transparent'. The user must feel free in unencumbered space (which requires a large and singularity free workspace, low inertia and low friction). He must also feel crisp contacts against the obstacles (which requires a sufficient force feedback, a high bandwidth and a large stiffness) [6] [7] [8].

These qualitative criteria are however not sufficient as existing input devices, which were all designed relying on them, exhibit very different performances [9] [10] [11] and are therefore more or less adapted to different applications. Precise requirements must thus be associated with each criterion.

Considering CAD like desktop applications, the ability of the user to perform quick and precise movements is of primary importance as it will directly affect its productivity. We will therefore use a stylus handle for dexterity and provide an elbow or wrist support for precision. The corresponding range of motions and efforts are around $150 \mathrm{~mm}$ and $10 \mathrm{~N}$ and $\pm 45^{\circ}$ and $0.25 \mathrm{~N} . \mathrm{m} \mathrm{[12]}$ (considering continuous force capacities). However, DC motors conventionally used on existing haptic devices exhibit a limited continuous torque compared to their high stall torque (with a ratio around 5). Therefore, to limit the weight of the device, we will limit the continuous force specifications to only $4 \mathrm{~N}$ and $0.1 \mathrm{~N}$.m. Considering the dynamic capacities of the motors, this will result in around $20 \mathrm{~N}$ and $0.5 \mathrm{~N} . \mathrm{m}$ peak force and torque as stated in Table 1.

Table 1. Desktop haptic interface design drivers

\begin{tabular}{|l|c|c|}
\hline & Translations & Rotations \\
\hline Range of motion & $150 \mathrm{~mm}$ & $\pm 45^{\circ}$ \\
\hline Peak force feedback & $20 \mathrm{~N}$ & $0.5 \mathrm{Nm}$ \\
\hline Cont. force feedback & $4 \mathrm{~N}$ & $0.1 \mathrm{Nm}$ \\
\hline
\end{tabular}




\section{Device architecture}

Most of existing master arms make use of four bar mechanisms serially connected to revolute joints either as a positioning stage if serial or as a sub-structure if parallel [12]. This simple and efficient solution allows to keep the motors close to the base thus reducing inertia. We will therefore limit our study to this type of structures and consider:

- serial structures called 1x6 as they exhibit 1 serial chain with 6 actuators. The wrist axes intersect either at the centre of the handle, allowing decoupled translations and rotations or above it, allowing a simplified and compact design.

- hybrid structures called $2 \times 3+1$ as they are made of two 5 DOFs / 3 motors robots connecting a fixed base to a platform carrying a $6^{\text {th }}$ actuated axis.

- $\quad$ parallel structures called $3 \times 2$ as they exhibit three 6 DOFs / 2 motors robots connecting the fixed base to a platform carrying the handle. It could be possible to use 3 actuators on each sub-structure to avoid singularities [14].

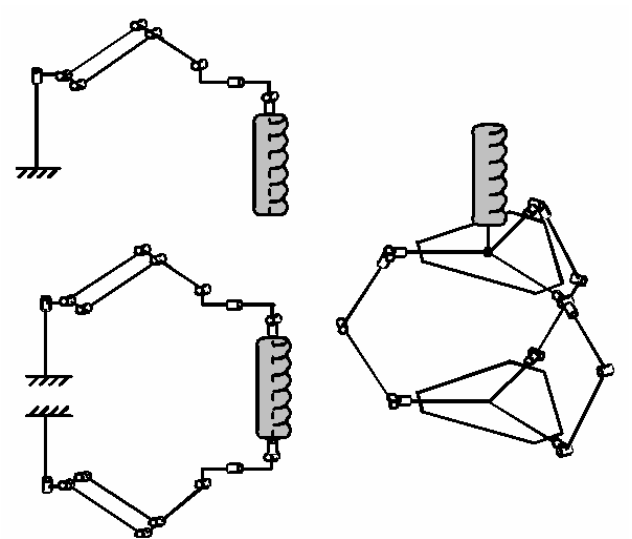

Fig. 1. Candidate architectures

We calculated for each architecture the maximum Homogeneous Orientation Workspace defined as the maximum rotation along the handle axis that can be reached for every inclination of the handle in a cone of the same angle value [12] (limited to $80^{\circ}$ which appears as a limit for the user). The HOW of $1 \times 6$ serial structures reaches $80^{\circ}$ while it is limited to $70^{\circ}$ to $80^{\circ}$ for $2 \times 3+1$ hybrid structures (depending on the configuration of the sub-structures which has an important influence on the collisions between the links) and to $40^{\circ}$ to $50^{\circ}$ in a reasonable portion of the total workspace for $3 \times 2$ parallel robots.
All these architectures are thus compatible with our design drivers. The $3 \times 2$ parallel structure will be favoured over serial or hybrid solutions as distal joint actuation would necessitate carried motors thus increasing inertia or complex transmissions (using cables and tendons [1]) thus increasing complexity and limiting the mechanical stiffness of the device.

Moreover, several advantages compared to existing parallel haptic devices [13] [14] will be introduced. The motors will be used to actuate the first and second axes of each sub-structure instead of the parallelogram axes (second and third axes) which is no more necessary, thus allowing a more compact design and less collisions between the links. Moreover, three of the six motors can be fixed on the base, thus reducing inertia. On the other hand, the sub-structures will be pointing towards the centre of the device in order to minimize its size (see Fig. 2). Finally, we will try to integrate the actuators in line with the links.

\section{Dimensioning and optimization}

\subsection{Design methodology}

Now that the architecture has been chosen, the new input device must be designed according to previous specifications. In this study, we will optimize the robot workspace as well as its static force efficiency. To reduce the complexity of such an optimization procedure, we will proceed in three steps:

- We will first make a preliminary dimensioning along with a first CAD design to choose the mains components and fix some limits on parameters.

- Then we will optimize the design in order to ensure force and torque capacities of $20 \mathrm{~N}$ and $0.5 \mathrm{~N} . \mathrm{m}$. The platform will be kept horizontal as taking all configurations into account would result in bulky actuation stages not compatible with a desktop set-up when considering cable capstan reducers which allow a high reversibility without the cost associated with extra force sensors.

- We will finally optimize the joints range of motion to avoid the large variety of singularities this architecture exhibits [14]. Therefore, mechanical stops will be introduced on the platform inclination (three extra motors could also be used but it would increase the complexity and cancel aforementioned advantages). Then we will be able to evaluate its performances when the handle is rotated. 


\subsection{Modeling tools}

The first design driver is the workspace of the robot defined as the set of valid configurations. To study this parameter, we scan the Cartesian space and use the inverse geometric model $q=g(X)$ to check which positions the robot can effectively reach.

The second design driver is the robot force capacity. To study this parameter, we use the notion of force ellipsoid defined as the operational forces produced by 1 N.m motor torques and described by the equation $F^{T} .\left(J_{m o t} \cdot J_{m o t}{ }^{T}\right) . F \leq 1$, with $J_{m o t}$ and $G_{m o t}$ the direct and inverse Jacobian matrices from motor to operational space. It allows to compute for each configuration the minimum force the robot can apply in all directions.

\subsection{Preliminary dimensioning}

The design of a robot must follow as much as possible the theoretical optimization results. However, the optimized parameters are often limited by technological or implementation constraints. In order to take them into account as soon as possible in the optimization process, we performed the theoretical dimensioning in parallel with the CAD design.

From preliminary results obtained after the study of a device with a base radius $r_{b}=150 \mathrm{~mm}$, sub-structures links lengths $1_{\mathrm{ss}}=100 \mathrm{~mm}$ and a platform radius $\mathrm{r}_{\mathrm{p}}=100 \mathrm{~mm}$ (see Fig. 2) which are compatible with a desktop set-up, we choose ironless DC motors whose continuous and stall torques are $112 \mathrm{mN} . \mathrm{m}$ and $493 \mathrm{mN} . \mathrm{m}$. From firsts CAD designs, we fixed the base radius $r_{b}=150 \mathrm{~mm}$ and the base height $h_{b}=100 \mathrm{~mm}$ in order to be able to integrate these components.

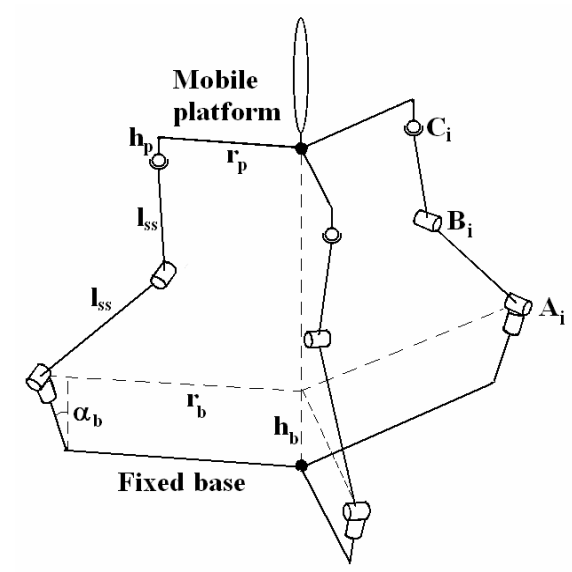

Fig. 2. Model of parallel haptic device

\subsection{First optimization step}

After previous study, we performed an optimization of the force and torque efficiency (during this first optimization step, the platform remains horizontal) with the constraints given in Table 1: the static capacities must be at least $20 \mathrm{~N}$ and $0.5 \mathrm{~N} . \mathrm{m}$ in every position of a $150 \mathrm{~mm}$ diameter sphere comprised in the workspace while the homogeneous orientation workspace must reach $\pm 45^{\circ}$. The parameters taken into account for this optimization are the following:

- The length of the links $1_{\mathrm{ss}}$ which vary between $100 \mathrm{~mm}$ and $200 \mathrm{~mm}$.

- The radius of the mobile platform $r_{p}$ which vary between $10 \mathrm{~mm}$ and $100 \mathrm{~mm}$.

- The inclination $\alpha_{b}$ of the first joint axis which vary between $0^{\circ}$ and $45^{\circ}$. It allows to reject the singularity occurring when the sub-structure end-tip crosses the first axis away from the workspace centre.

- The height of the workspace centre (for each height value, we compute the largest sphere diameter included in the total workspace of the robot and compare it with the design drivers).

The optimal dimensions are $\alpha_{\text {bopt }}=40^{\circ}, 1_{\text {ssopt }}=120 \mathrm{~mm}$ and $\mathrm{r}_{\mathrm{popt}}=50 \mathrm{~mm}$ and the optimal workspace centre height is $230 \mathrm{~mm}$ above the base. In this case, the reduction ratios reach their minimal value. They are limited to 11.1 and 4.6 on axes 1 and 2, which is fully compatible with compact cable capstan reducers.

\subsection{Second optimization step}

The $3 \times 2$ parallel architecture exhibits a large variety of singularities, some of which appear within the useful workspace of the robot when the platform moves in 6 DOF. To avoid these singular configurations, we will introduce mechanical stops on the joints of the robot:

- The range of motion of the wrist axes will limit the homogeneous orientation workspace to $45^{\circ}$ at the centre of the workspace as stated in Table 1.

- The range of motion of the three first axes will limit the useful workspace to a $150 \mathrm{~mm}$ sphere.

The homogeneous orientation workspace obtained taking these limitations into account is illustrated by Fig. 3 as a function of the position of the platform and as a function of the distance from the centre of the useful workspace. These results show that the total workspace of the robot is much larger than its useful workspace which is a $150 \mathrm{~mm}$ diameter sphere. Moreover, the homogeneous orientation workspace is $\pm 45^{\circ}$ at the centre of the 
workspace while it remains over $\pm 30^{\circ}$ in a $100 \mathrm{~mm}$ diameter sphere.
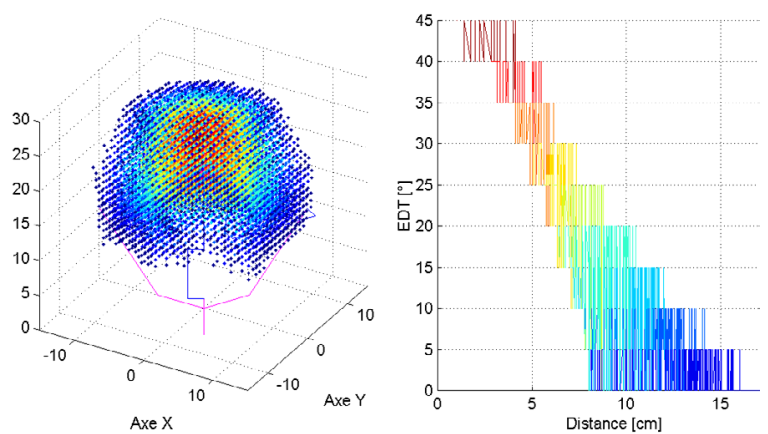

Fig. 3. Homogeneous orientation workspace
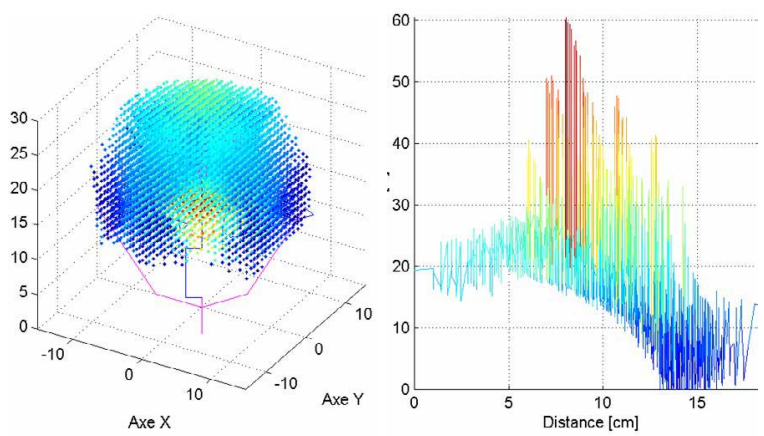

Fig. 4. Static peak force capacity

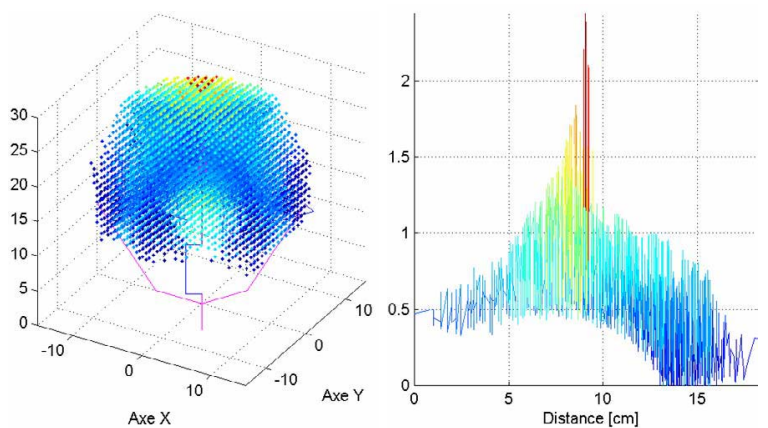

Fig. 5. Static peak torque capacity

The minimum amount of peak force and torque the robot can apply in any direction considering all the possible configurations within the homogeneous orientation workspace are illustrated by Fig. 4 and 5 . These figures show that even if only horizontal platform configurations were considered for actuators dimensioning, the peak force and torque capacities are above $20 \mathrm{~N}$ and $0.5 \mathrm{~N} . \mathrm{m}$ in the centre of the workspace as stated in Table 1 and remains over $14.3 \mathrm{~N}$ and $0.32 \mathrm{~N} . \mathrm{m}$ in the $150 \mathrm{~mm}$ diameter useful workspace even when considering the worst case obtained in the homogeneous orientation workspace which is up to $\pm 45^{\circ}$ at the centre of the workspace. In fact, the robot actuators are dimensioned in horizontal platform configurations in order to ensure simultaneous force and torque capacities above $20 \mathrm{~N}$ and $0.5 \mathrm{~N} . \mathrm{m}$. When considering only force or torque, the capacities are well above and they remain around our design drivers even when considering the worst case over the local homogeneous orientation workspace.

\section{Final design}

The final CAD design of the device is illustrated by Fig. 6. It emphasizes ironless DC motors and cable capstan reducers with 11.1 and 4.6 reduction ratios as stated in sections 4.3 and 4.4. First axis is inclined $40^{\circ}$ away from the vertical and links lengths equal $120 \mathrm{~mm}$ as stated in section 4.4. Finally, mechanical stops are introduced on the joints of the robot as stated in section 4.5 .

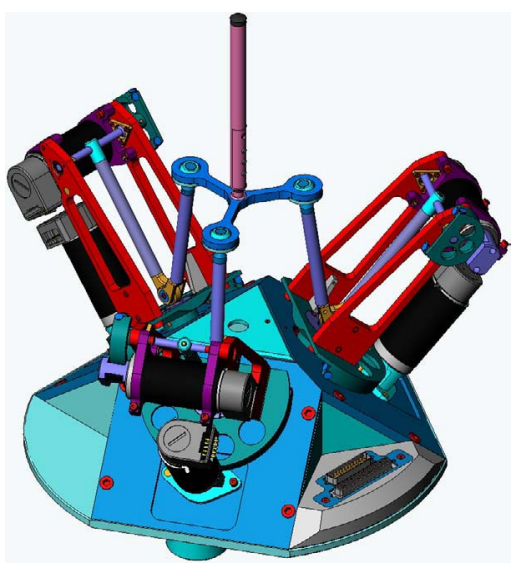

Fig. 6. 3x2 parallel haptic interface CAD design

\section{Control}

The interface controller was implemented in the Haption company generic controller, running under linux RTAI (www.haption.com).

The principle of the control scheme is the same as used for remote handling [15]. It is based on a Cartesian impedance control and requires the forward kinematics function $X_{\text {platform }}=f\left(\theta_{m}\right)$ and the forward geometric jacobian $J_{m o t}$ to be computed (see Fig. 7). 


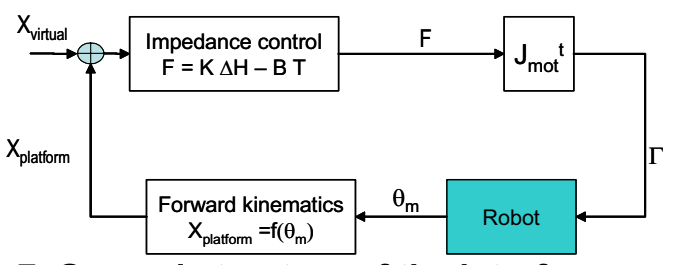

Fig. 7. General structure of the interface control

\subsection{Forward kinematics}

The forward kinematics function determines the platform position $X_{\text {platform }}$ from the 6 active joints displacements $\theta_{m}$, measured by the motors encoders.

Let us consider one of the 3 serial sub-structures $i$ $(i=1,3)$ (see Fig. 2). The center $C_{i}$ of the passive spherical joint could be easily determined from the displacements of the three revolute joints, then $X_{\text {platform }}$ from the position of the end points of the 3 legs $\left(\mathrm{C}_{1}, \mathrm{C}_{2}, \mathrm{C}_{3}\right)$. However, the motor encoders only measure the displacements of the first two joints. To get a simple forward kinematics function, an extra position sensor would be necessary [16]. This solution was however rejected for cost and maintainability reasons.

A numerical iterative solution is then required to solve the forward kinematics problem. This problem is reduced here to a 3-dimensional inversion for determining the passive revolute joints displacements $\underline{\theta}_{p}$ from the active ones $\underline{\theta}_{a}$. The mapping to be inverted $\underline{l}=\underline{l}\left(\underline{\theta}_{a}, \theta_{p}\right)$ express the closure of the three-legs on the platform as $\left\|\underline{C}_{i} \underline{C}_{j}\right\|=l_{i j}$ where $\underline{l}=\left(l_{12}, l_{23}, l_{31}\right)^{T}$ is the vector of the three lengths of the platform triangle.

The differential relationship $\underline{\dot{i}}=J_{l a} \cdot \underline{\dot{\theta}}_{a}+J_{l p} \cdot \underline{\dot{\theta}}_{p}$, whose derivation is detailed in [17], may be used to write an iterative numerical solution. Yang and al. [16] use a Newton-Raphson algorithm for geometric simulation purpose, with inversion of matrix $J_{l p}$. To ensure stability of the numerical inversion included in the overall haptic system, we modify this numerical scheme based on passive control schemes.

\subsection{Forward geometric Jacobian}

The geometric Jacobian $G_{m o t}$ relates the wrench of external forces on the platform $\underline{W}=G_{m o t}{ }^{T} \cdot \underline{\tau}_{a}$ to the active torques $\tau_{a} \cdot \underline{W}=(\underline{F}, \underline{m}(O))^{T}$ is the Plûcker coordinates of the wrench, where $\underline{F}$ and $\underline{m}(O)$ are the force vector and the momentum of forces at point $\mathrm{O}$, in the fixed reference frame. The columns of $G_{m o t}{ }^{T}$ are the Plücker coordinates of line-vectors called the wrench supports of the active joints.
Let us consider one of the leg $i$ and its 2 active joints. This leg applies on the platform a wrench $\underline{W}_{i}$ which is a linear combination of the two active joints torques $\tau_{i l}$ and $\tau_{i 2}: \underline{W}_{i}=\underline{L_{i 1}} . \tau_{i 1}+\underline{L_{i 2}} . \tau_{i 2}$.

The wrench supports $\underline{L}_{i 1}$ and $\underline{L}_{i 2}$ are geometrically determined using reciprocity and duality relationships between the wrench supports and the twists associated with the 6 joints of the leg, considering active and passive ones. This method relying on vector space calculus and geometry of screws and lines is presented in [18]. We obtain thus $\underline{\mathrm{L}}_{\mathrm{i} 1}=\underline{\mathrm{L}}_{\mathrm{C} \mu \mathrm{i}} / \mu \mathrm{i}$ and $\underline{\mathrm{L}}_{\mathrm{i} 2}=\underline{\mathrm{L}}_{\mathrm{BiC}} / \lambda \mathrm{i}$ with :

- $\underline{L}_{C \mu i}=\left(\underline{u}_{i}, O C_{i} \times \underline{u}_{i}\right)^{T}$ the Plücker coordinates of the line passing through $C_{i}$, the end-point of the leg, and directed by unit vector $\underline{u}_{i}$ representing the direction of the two parallel revolute joints of the leg $\mathrm{i}$,

- $\underline{L}_{B i C}=\left(\underline{t}_{i}, \underline{O B_{i}} \times \underline{t}_{i}\right)^{T}$ the Plücker coordinates of the line passing through points $B_{i}$ and $C_{i}$, with direction unit vector $\underline{t}_{i}=\underline{B}_{i} \underline{C}_{i}\left\|\underline{B}_{i} \underline{C}_{i}\right\|$,

- $\mu i=\underline{A}_{i} \underline{C_{i}} \cdot \underline{n_{i}}$ and $\lambda i=\underline{A}_{i} \underline{B}_{i} \cdot\left(\underline{t}_{i} \times \underline{u}_{i}\right)$

The matrix $G_{m o t}^{T}=\left[\begin{array}{llllll}\underline{L}_{11} & \underline{L}_{12} & \underline{L}_{21} & \underline{L}_{22} & \underline{L}_{31} & \underline{L}_{32}\end{array}\right]$ is thus composed of a basis of 6 line-vectors. Its inversion is equivalent to the determination of its dual basis. As the 2 axes of each pair of line-vectors $\left(\underline{L}_{i l}, \underline{L}_{i 2}\right)$ associated with leg i pass through a common point $C_{i}$ (see Fig. 8), this geometric configuration of wrench supports is similar to those of a 6-3 Stewart platform. Taking advantage of this special geometry, the inversion problem may be solved in closed form with very basic line geometry calculations and reduces to the inversion of three 2 dimensional matrices, one for each leg. This geometric inversion is detailed in section III-3 of [18].

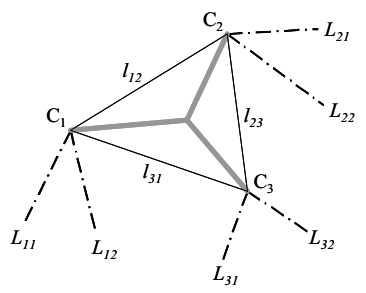

Fig. 8. Geometric configuration of the 6 wrench supports

\section{Integration and tests}

The $3 \times 2$ parallel haptic device was integrated in CAD applications (see Fig. 9). The user manipulates the virtual objects via a stylus handle incorporating 3 selection buttons (grasp - release objects, clutch - declutch, the third one being re-configurable). 


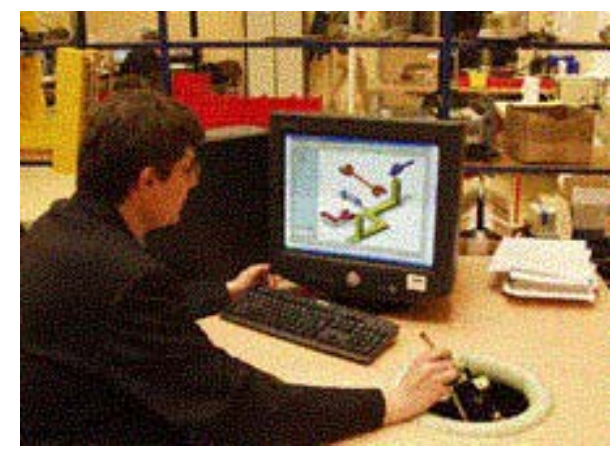

Fig. 9. 3x2 parallel haptic interface

It emphasizes a sufficient workspace for desktop manipulation (arm or wrist supported) and the force feedback allows to recreate a realistic virtual world. The user is able to select virtual objects, to catch them and to make virtual assembly with force feedback.

\section{Conclusion}

In this article, we presented the design and optimization of a novel desktop 6 DOF parallel haptic device successfully coupled to CAD applications.

Future works concern device parameters and performances identification as well as manipulation in real usage situation in a haptics user lab centered amongst other on CAD applications.

\section{References}

[1] J. Vertut, C. Coiffet, "Teleoperation and Robotics Evolution and Development", Robot Technology Volume 3A, Hermès Publishing, Paris, 1985.

[2] C. Andriot, "Automatique des systèmes téléopérés avec retour d'effort, limitation des performances", Ph.D. diss. (in French), University of Paris 6, Octobre 1992.

[3] F. Gosselin, A. Riwan, "Design of VIRTUOSE 3D : a new haptic interface for teleoperation and virtual reality", Proc. $10^{\text {th }}$ Int. Conf. on Advanced Robotics, August 22-25, 2001, Budapest, Hungary, pp.205-212.

[4] F. Gosselin, A. Riwan, D. Ponsort, J.P. Friconneau, P. Gravez, "Design of a new input device for telesurgery", World Automation Congress 2004, Proc. $10^{\text {th }}$ Int. Symp. on Robotics and Applications, June 28-July 1, 2004, Seville, Spain, Paper ISORA 120.

[5] F. Gosselin, T. Jouan, J. Brisset, C. Andriot, "Design of a wearable haptic interface for precise finger interactions in large virtual environments", submitted to World Haptics, Pisa, Italy March 18-20, 2005.
[6] T.H. Massie, J.K. Salisbury, "The PHANToM haptic interface: a device for probing virtual objects", Proc. ASME Winter Annual Meeting, Symp. on Haptic Interfaces for Virtual Environment and Teleoperator Systems, Chicago, November 1994.

[7] H. Iwata, "Force Display for Virtual Worlds", Proc. Int. Conf. on Artificial Reality and Tele-existence, Tokyo, Japan, 1991, pp. 111-116.

[8] R. Baumann, R. Clavel, "Haptic interface for virtual reality based minimally invasive surgery simulation", Proc. IEEE Int. Conf. on Robotics and Automation, Louvain, Belgium, May 1998, pp. 381-386.

[9] D.A. McAffee, P. Fiorini, "Hand Controller Design Requirements and Performance Issues inTelerobotics", ICAR 91, Robots in Unstructured Environments, Pisa, Italy, June 1991, pp. 186-192.

[10] G.W. Köhler, “Typenbuch der Manipulatoren Manipulator Type Book”, Thiemig Taschenbücher, Verlag Karl Thiemig, München, 1981.

[11] G. Burdea, P. Coiffet, "La réalité virtuelle", Hermès Publishing, Paris, 1993.

[12] F. Gosselin, “Développement d'outils d'aide à la conception d'organes de commande pour la téléopération à retour d'effort", Ph.D. diss. (in French), University of Poitiers, June 2000.

[13] M.H. Brown et J.D. Asquith, "A Man-Machine Interface With Feeling”, Service Robot, Vol. 2, 1996.

[14] G.L. Long, C.L. Collins, "A Pantograph Linkage Parallel Platform Master Hand Controller for Force-Reflexion", Proc. IEEE Int. Conf. on Robotics and Automation, Nice, France, May 1992.

[15] A. Micaelli, "Asservissements et lois de couplage en téléopération", in Téléopération et télérobotique. ed. P.Coiffet A.Kheddar, Hermes edition, 2002.

[16] G. Yang, I.-M. Chen, W.K. Lim, S.H. Yeo, "SelfCalibration of Three-Legged Modular Reconfigurable Parallel Robots Using Leg-End Distance Errors", Robotica, Vol. 19, pp. 187-198, 2001.

[17] G. Yang, I.-M. Chen, W. Lin, J. Angeles, "Singularity Analysis of Three-Legged Parallel Robots Based on Passive-Joints Velocities", IEEE Trans. on Robotics and Automation, vol. 17, No. 4, pp. 413-422, August 2001.

[18] C. Bidard, "Dual bases of screw-vectors for inverse kinestatic problems in robotics", in Advances in Robot Kinematics and Computational Geometry, ed. J.Lenarcic B.Ravani, pp. 339-348, Kluver Academic Press, 1994. 